\title{
Sand Piles Models of Signed Partitions with $d$ Piles
}

\author{
C. Bisi, ${ }^{1}$ G. Chiaselotti, ${ }^{2}$ and P. A. Oliverio ${ }^{2}$ \\ ${ }^{1}$ Dipartimento di Matematica e Informatica, Universitá di Ferrara, \\ Via Machiavelli 35, 44121 Ferrara, Italy \\ ${ }^{2}$ Dipartimento di Matematica, Universitá della Calabria, Via Pietro Bucci, \\ Cubo 30B, 87036 Arcavacata di Rende, Italy \\ Correspondence should be addressed to G. Chiaselotti; chiaselotti@unical.it
}

Received 3 October 2012; Accepted 4 November 2012

Academic Editors: J. F. Fang, N. A. Gordon, and M.-J. Jou

Copyright (c) 2013 C. Bisi et al. This is an open access article distributed under the Creative Commons Attribution License, which permits unrestricted use, distribution, and reproduction in any medium, provided the original work is properly cited.

Let $r, d \leq n$ be nonnegative integers. In this paper we study the basic properties of a discrete dynamical model of signed integer partitions that we denote by $S(n, d, r)$. A generic element of this model is a signed integer partition with exactly $d$ all distinct nonzero parts, whose maximum positive summand is not exceeding $r$ and whose minimum negative summand is not less than $-(n-r)$. In particular, we determine the covering relations, the rank function, and the parallel convergence time from the bottom to the top of $S(n, d, r)$ by using an abstract Sand Piles Model with three evolution rules. The lattice $S(n, d, r)$ was introduced by the first two authors in order to study some combinatorial extremal sum problems.

\section{Introduction}

Discrete dynamical models whose configurations are integer partitions are also called Sand Piles Models and they have been deeply investigated. In these models an integer partition is treated as a sequence of piles of grains of sand and each singular grain as a single integer unit. An evolution rule in these models is a rule which describes how to move some particular grains of a configuration in order to obtain another configuration. The famous Brylawski paper [1] can be considered the first implicit study of an integer partitions lattice by means of two evolution dynamical rules which determine the covering relations of this lattice. In [1] Brylawski proposed a dynamical approach to study the lattice $L_{B}(n)$ of all the partitions of a fixed positive integer $n$ with the dominance order.

However, the explicit identification of a specific set of integer partitions with a Sand Piles Model begins in $[2,3]$.

In the Sand Piles Model introduced by Goles and Kiwi in [3], denoted by $\operatorname{SPM}(n)$, a sand pile is represented by an ordered partition of an integer $n$, that is, a decreasing sequence $a=\left(a_{1}, \ldots, a_{n}\right)$ having sum $n$, and the movement of a sand grain respects the following rule.

Rule 1 (vertical rule). One grain can move from a column to the next one if the difference of height of these two columns is greater than or equal to 2 .

In the model $L_{B}(n)$ (introduced by Brylawski, 1973 [1]), the movement of a sand grain respects Rule 1 and Rule 2, which is described as follows.

Rule 2 (horizontal rule). If a column containing $p+1$ grains, is followed by a sequence of columns containing $p$ grains and then one column containing $p-1$ grains, one grain of the first column can slip to the last one.

The Sand Piles Model SPM $(n)$ is a special case of the more general Chip Firing Game (CFG), which was introduced by Spencer in [4] to study some "balancing game". There are a lot of specializations and extensions of this model which have been introduced and studied under different names, different aspects and different approaches. The $\operatorname{SPM}(n)$ can 
be also related to the Self-Organized Criticality (SOC) system introduced by Bak et al. in [5]. The study of such systems have been developed in an algebraic context ([6]), in a combinatorial games theory setting $([3,7,8])$ and in the theory of cellular automata $[9,10]$.

In the papers $[8,11-20]$, several dynamical models related to $\operatorname{SPM}(n)$ have been studied. Almost all systems studied in the previous works have a linear topology and they have extended the classical models $\operatorname{SPM}(n)$ and $L_{B}(n)$ to obtain more general models. An excellent survey on these topics is [21].

Let now $r$ and $d$ be non-negative integers less or equal than $n$ and $[n]$ the $n$-set $\{1, \ldots, n\}$. In [22] the authors have introduced and studied a poset $(S(n, r)$, ㄷ) related to some extremal combinatorial sums problems (see also [2326] for studies on these problems). Such a poset can be seen as a lattice of particular integer partitions with all distinct summands which can be positive or negative, whose maximum positive summand is not exceeding $r$ and whose minimum negative summand is not less than $-(n-r)$. Such a poset is an involution poset; that is, it has an involution map that gives it some special symmetric properties, and it is also a lattice isomorphic (as noted in [27]) to the direct product $M(r) \times M(n-r)^{*}$, where $M(n)$ is the lattice introduced by Stanley, [28], in order to solve an Erdös and Moser conjecture (here we denote by $M(n, r)^{*}$ the dual lattice of $\left.M(n-r)\right)$. The structure of $M(n)$ is well known. For example, since $M(n)$ is Peck, it follows that $S(n, r)$ is Peck by [29]. This lattice contains an interesting sublattice $(S(n, d, r)$, ㄷ) that is the set of all the integer partitions of $S(n, r)$ having exactly $d$ non-zero summands. Also this sublattice has been introduced in [22] and its structure at present is incompletely understood. For example, we know that it is a graded poset because it is a finite distributive lattice, but its rank function is unknown. In [30] Andrews introduced the concept of signed partition: a signed partition is a finite sequence of integers $\lambda_{k}, \ldots, \lambda_{1}, \lambda_{-1}, \ldots, \lambda_{-l}$ such that $\lambda_{k} \geq \cdots \geq \lambda_{1}>0>\lambda_{-1} \geq \cdots \geq \lambda_{-l}$. In [30, 31] the signed partitions are studied from an arithmetical point of view.

In this paper we study the lattice $S(n, d, r)$ as a Sand Piles Model of signed integer partitions with three evolution rules. The first of these rules is an outside adjunction rule on the "positive" piles. The second rule is a switching rule between "negative" piles and "positive" piles which allows to maintain constant the number of the piles. The third rule is an outside elimination rule on the negative "piles". We prove that the covering relation in the lattice $S(n, d, r)$ is uniquely determined from the three previous rules. The paper is articulated as follows. In Section 1 we recall some basic definitions and preliminary results, for example, the definition of $S(n, d, r)$ and some of its properties. In Section 2 we explain how to see the signed partitions of $S(n, d, r)$ as configurations of our Sand Piles Model and also we describe its evolution rules. In Section 3 we prove (Theorem 3 ) that the covering relation in the lattice $S(n, d, r)$ is uniquely determined by three evolution rules of our Sand Piles Model. We determine the rank function of $S(n, d, r)$ and we compute the rank of $S(n, d, r)$, that is, the sequential convergence time from the minimum to the maximum in $S(n, d, r)$. Finally, in
Section 4 we give some estimates for the parallel convergence time in our model.

\section{Definitions and Preliminary Results}

If $(X, \leq)$ is a poset and $x, y \in X$, we write $y>x$ (or $x<$ $y$ ) if $y$ covers $x$. Now we briefly recall the definition of the lattice $S(n, r)$ that we have introduced in [22] in a more formal context. In this paper we always denote with $n$ and $r$ two fixed non-negative integers such that $r \leq n$. We call $(n, r)$-string an $n$-pla of integers

$$
a_{r} \ldots a_{1} \mid b_{1} \ldots b_{n-r}
$$

such that

(i) $a_{1}, \ldots, a_{r} \in\{1, \ldots, r, 0\}$;

(ii) $b_{1}, \ldots, b_{n-r} \in\{-1, \ldots,-(n-r), 0\}$;

(iii) $a_{r} \geq \cdots \geq a_{1} \geq 0 \geq b_{1} \geq \cdots \geq b_{n-r}$;

(iv) the unique element in (1) which can be repeated is 0 .

If $w$ is a $(n, r)$-string, we call parts of $w$ the integers $a_{r}$, $\ldots, a_{1}, b_{1} \ldots b_{n-r}$, non-negative parts of $w$ the integers $a_{r}, \ldots, a_{1}$ and non-positive parts of $w$ the integers $b_{1} \ldots b_{n-r}$. We set $\sum(w):=\sum_{i=1}^{r} a_{i}+\sum_{j=1}^{n-r} b_{j}$, and if $m \in \mathbb{Z}$ is such that $\sum(w)=m$, we say that $w$ is a signed partitions of $m$; in this case we write $w \vdash m$. We set $w_{+}=a_{r} \ldots a_{1} \mid$ and $w_{-}=\mid$ $b_{1} \ldots b_{n-r}$. Also, we denote by $|w|_{>}$the number of parts of $w$ that are strictly positive, with $|w|_{<}$the number of parts of $w$ that are strictly negative and we set $\|w\|=|w|_{>}+|w|_{<.} S(n, r)$ is the set of all the $(n, r)$-strings. If $w=a_{r} \ldots a_{1} \mid b_{1} \ldots b_{n-r}$ and $w^{\prime}=a_{r}^{\prime} \ldots a_{1}^{\prime} \mid b_{1}^{\prime} \ldots b_{n-r}^{\prime}$ are two $(n, r)$-strings, we set $w_{+}=w_{+}^{\prime}$ if $a_{i}^{\prime}=a_{i}$ for all $i=r, \ldots, 1, w_{-}=w_{-}^{\prime}$ if $b_{j}^{\prime}=b_{j}$ for all $j=1, \ldots, n-r$ and $w=w$ if $w_{+}=w_{+}^{\prime}$ and $w_{-}=w_{-}^{\prime}$. On $S(n, r)$ we consider the partial order on the components, that we denote by $\sqsubseteq$. To simplify the notations, in all the numerical examples the integers on the right of the vertical bar | will be written without minus sign. Since $(S(n, r), \sqsubseteq)$ is a finite distributive lattice it is also graded, with minimum element $0 \cdots 0 \mid 12 \cdots(n-r)$ and maximum element $r(r-1) \cdots 21 \mid$ $0 \cdots 0$.

We recall now the concept of involution poset (see [32, 33] for some recent studies on such class of posets). An involution poset (IP) is a poset $(X, \leq, c)$ with a unary operation $c: x \in$ $X \mapsto x^{c} \in X$, such that

(I1) $\left(x^{c}\right)^{c}=x$, for all $x \in X$;

(I2) if $x, y \in X$ and if $x \leq y$, then $y^{c} \leq x^{c}$.

The map $c$ is called complementation of $X$ and $x^{c}$ the complement of $x$. Let us observe that if $X$ is an involution poset, by (I1) follows that $c$ is bijective and by (I1) and (I2) it holds that if $x, y \in X$ are such that $x<y$, then $y^{c}<x^{c}$. If $(X, \leq, c)$ is an involution poset and if $Z \subseteq X$, we will set $Z^{c}=\left\{z^{c}: z \in Z\right\}$. We note that if $X$ is an involution poset then $X$ is a self-dual poset because from (I1) and (I2) it follows that if $x, y \in X$ we have that $x \leq y$, if and only if $y^{c} \leq$ $x^{c}$, and this is equivalent to say that the complementation is 
an isomorphism between $X$ and its dual poset $X^{*}$. In [22] has been shown that $(S(n, r), \sqsubseteq)$ is an involution poset and its complementation map $c$ is the following:

$$
\begin{aligned}
& \left(\begin{array}{llllllll}
a_{k} \ldots & a_{1} & 0 \ldots 0 \mid 0 & \ldots 0 & \left.b_{1} \ldots b_{l}\right)^{c} \\
& =a_{r-k}^{\prime} \ldots a_{1}^{\prime} & 0 \ldots 0 \mid & \ldots & \ldots 0 & b_{1}^{\prime} \ldots b_{n-r-l}^{\prime},
\end{array}\right.
\end{aligned}
$$

where $\left\{a_{1}^{\prime}, \ldots, a_{r-k}^{\prime}\right\}$ is the usual complement of $\left\{a_{1}, \ldots, a_{k}\right\}$ in $\{1, \ldots, r\}$, and $\left\{b_{1}^{\prime}, \ldots, b_{n-r-l}^{\prime}\right\}$ is the usual complement of $\left\{b_{1}, \ldots, b_{l}\right\}$ in $\{-1, \ldots,-(n-r)\}$ (e.g., in $S(7,4)$, we have that $\left.(4310 \mid 001)^{c}=2000 \mid 023\right)$. If $d$ is an integer such that $0 \leq d \leq n$, we set now $S(n, d, r)=\{w \in S(n, r):\|w\|=d\}$. It's easy to see that $(S(n, d, r)$, ᄃ) is a sub-lattice of $(S(n, r)$, $\sqsubseteq)$ and obviously $|S(n, d, r)|=\left(\begin{array}{l}n \\ d\end{array}\right)$. In the sequel we always denote, respectively, by $\widehat{0}$ and $\widehat{1}$ the minimum and the maximum element of the lattice $S(n, d, r)$.

\section{Evolution Rules}

In this section we describe a discrete dynamical model with three evolution rules. In this model a configuration will be a generic element of $S(n, d, r)$. In the sequel, to comply with the terminology concerning the Sand Piles Models, if $w \in$ $S(n, d, r)$, we represent the sequence of the positive parts of $w$ as a not-increasing sequence of columns of stacked squares and the sequence of the negative parts of $w$ as a notdecreasing sequence of columns of stacked squares. We call a column of stacked squares a pile and each square of a pile is called a grain. For example, if $n=10, r=6, d=5$, the configuration

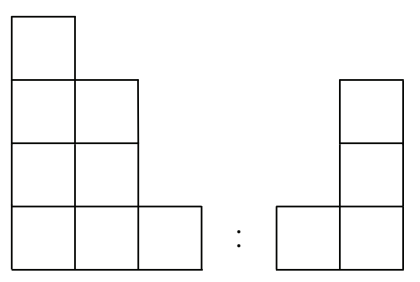

is identified with the partition $(4,3,1,0,0,0 \mid 0,0,-1,-3)=$ $433100 \mid 0113 \in S(10,5,6)$. We denote by $D(w):=D^{+}(w)$ : $D^{-}(w)$ the configuration associated to $w$, where $D^{+}(w)$ is the Young diagram (represented with not-increasing columns) of the partition $\left(a_{r}, \ldots, a_{1}\right)$ and $D^{-}(w)$ is the Young diagram (represented with not-decreasing columns) of the partition with negative summands $\left(-b_{1}, \ldots,-b_{n-r}\right)$. Our goal is to define some rules of evolution that starting from the minimum of $S(n, d, r)$ allow us to reconstruct the Hasse diagram of $S(n, d, r)$ (and therefore to determine the covering relations in $S(n, d, r))$.

Let $w=a_{r} \ldots a_{1} \mid b_{1} \ldots b_{n-r} \in S(n, d, r)$. We formally set $a_{0}:=0, a_{r+1}:=r+1$ and $b_{0}:=0$. If $0 \leq i \leq r+1$ we call $a_{i}$ the ith-plus pile of $w$, and if $0 \leq j \leq n-r$ we call $b_{j}$ the $j$ th-minus pile of $w$. We call $a_{i}$ plus singleton pile if $a_{i}=1$ and $b_{j}$ minus singleton pile if $b_{j}=-1$. If $1 \leq i \leq r+1$ we set $\Delta_{i}^{+}(w)=a_{i}-a_{i-1}$ and we call $\Delta_{i}^{+}(w)$ the plus height difference of $w$ in $i$. If $1 \leq j \leq n-r$ we set $\Delta_{j}^{-}(w)=\left|b_{j}\right|-\left|b_{j-1}\right|$ and we call $\Delta_{j}^{-}(w)$ the minus height difference of $w$ in $j$. If $1<i \leq r$, we say that $w$ has a plus cliff at $i$ if $\Delta_{i}^{+}(w) \geq 2$. If $1<j \leq n-r$, we say that $w$ has a minus cliff at $j$ if $\Delta_{j}^{-}(w) \geq 2$.

Remark 1. The choice to set $a_{0}=0, a_{r+1}=r$ and $b_{0}=0$ is a formal trick for decrease the number of rules necessary for our model. This means that when we apply the next rules to one element $w \in S(n, d, r)$ we think that there is an "invisible" extra pile in the imaginary place $r+1$ having exactly $r+1$ grains, an "invisible" extra pile with 0 grains in the imaginary place to the right of $a_{1}$ and to the left of $\mid$ and another "invisible" extra pile with 0 grains in the imaginary place to the left of $b_{1}$ and to the right of $\mid$. However the piles corresponding respectively to $a_{0}=0, a_{r+1}=r+1$ and $b_{0}=0$ must be not considered as parts of $w$.

\subsection{Evolution Rules}

$R_{1}$ : If the $i$ th-plus pile has at least one grain and if $w$ has a plus cliff at $i+1$ then one grain must be added on the ith-plus pile:
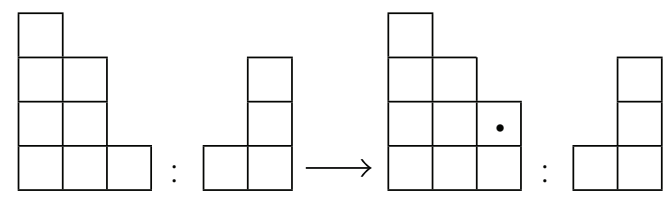

$R_{2}$ : If there is not a plus singleton pile and there is a minus singleton pile, then the latter must be shifted to the side of the lowest not empty plus pile:
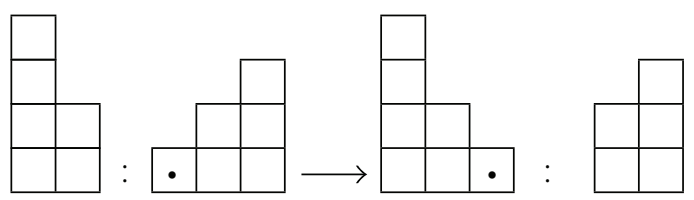

$R_{3}$ : One grain must be deleted from the $j$ th-minus pile if $w$ has a minus cliff at $j$ :
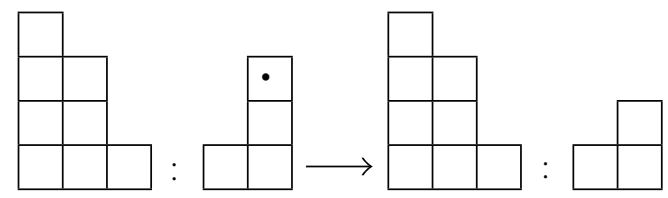

Remark 2. (i) Under the hypothesis in $R_{3}$, the $j$ th minus pile must have at least 2 grains.

(ii) In $R_{2}$ the lowest not empty plus pile can also be the invisible column in the place $r+1$. In this case all the plus piles are empty and an eventual minus singleton pile must be shifted in the place $r$.

\section{Covering Relations in $S(n, d, r)$}

In this section we describe the covering relation in the lattice $S(n, d, r)$. The main result of this section is the Theorem 3 . In the sequel we write $w \rightarrow{ }^{k} w^{\prime}$ (or $w^{\prime}=w \rightarrow^{k}$ ) to denote 
that $w^{\prime}$ is a $n$-pla of integers obtained from $w$ applying $R_{k}$, for $k=1,2,3$.

Theorem 3. (i) If $w \in S(n, d, r)$ and $w^{\prime}=w \rightarrow{ }^{k}$ for some $k=1,2,3$, then $w^{\prime} \in S(n, d, r)$ and $w^{\prime}>w$.

(ii) If $w, w^{\prime} \in S(n, d, r)$ and $w^{\prime}>w$, then $w^{\prime}=w \rightarrow{ }^{k}$ for some $k=1,2,3$.

Proof. (i) Let $w=a_{r} \ldots a_{1} \mid b_{1} \ldots b_{n-r} \in S(n, d, r), a_{r+1}=$ $r+1$ (the invisible pile in the place $r+1$ ) and $D=D(w)$. We distinguish the three possible cases related to the previous rules.

Case 1. Let us assume that $r \geq i \geq 1, a_{i} \neq 0$ and that $w$ has a plus cliff at $i+1$. If $w^{\prime}=w \rightarrow{ }^{1}$, then $w^{\prime}=a_{r} \ldots a_{i+1}\left(a_{i}+\right.$ 1) $a_{i-1} \ldots a_{1} \mid b_{1} \ldots b_{n-r}$. It is clear that $\left\|w^{\prime}\right\|=d$ because $a_{i} \neq 0$. Since there is a plus cliff at $i+1$ we have $a_{i+1}-a_{i} \geq 2$, hence $a_{i+1} \geq a_{i}+2>a_{i}+1>a_{i}>a_{i-1}$, and this implies that $w^{\prime} \in S(n, d, r)$. We must show now that $w^{\prime}$ covers $w$ in $S(n, d, r)$. Since $w$ and $w^{\prime}$ differ between them only in the place $i$ for $a_{i}$ and $a_{i}+1$, respectively, it is clear that there does not exist an element $z \in S(n, d, r)$ such that $w \sqsubset z \sqsubset w^{\prime}$. Hence $w^{\prime}>w$.

Case 2. Let us assume that in $D$ there is not a plus singleton pile and that there is a minus singleton pile $b_{j}=-1$ for some $1 \leq j \leq n-r$. Since $a_{r+1}=r+1$, we can assume that $a_{i+1}>0, a_{i}=0$, for some $1 \leq i \leq r$. This means that $w$ has the following form: $w=a_{r} \ldots a_{i+1} 00 \ldots 0$ | $0 \ldots 0(-1) b_{j+1} \ldots b_{n-r}$, where $a_{i+1}>1$ (otherwise $D$ has a plus singleton pile). Applying $R_{2}$ to $w$ we obtain $w^{\prime}=w \rightarrow^{2}$, where $w^{\prime}=a_{r} \ldots a_{i+1} 10 \ldots 0 \mid 0 \ldots 00 b_{j+1} \ldots b_{n-r}$. It is clear then that $w^{\prime} \in S(n, r)$ and $\left\|w^{\prime}\right\|=d$ since $w^{\prime}$ is obtained from $w$ with only a shift of the pile -1 to the left in the place $i$. Let us note that the only elements $z_{1}, z_{2} \in$ $S(n, r)$ such that $w \sqsubset z_{1} \sqsubset w^{\prime}$ and $w \sqsubset z_{2} \sqsubset w^{\prime}$ are $z_{1}=a_{r} \ldots a_{i+1} 10 \ldots 0 \quad 0 \ldots 0(-1) b_{j+1} \ldots b_{n-r}$ and $z_{2}=$ $a_{r} \ldots a_{i+1} 00 \ldots 0 \mid 0 \ldots 00 b_{j+1} \ldots b_{n-r}$, but $\left\|z_{1}\right\|=d+1$ and $\left\|z_{2}\right\|=d-1$, hence $z_{1}, z_{2}$ are not elements of $S(n, d, r)$. This implies that $w^{\prime}$ covers $w$ in $S(n, d, r)$.

Case 3. If $1<j \leq n-r$ and $w$ has a minus cliff at $j$, we apply $R_{3}$ to $w$ on the pile $b_{j}$ and we obtain $w^{\prime}=w \rightarrow^{3}$, where $w^{\prime}=a_{r} \ldots a_{1} \mid b_{1} \ldots b_{j-1}\left(b_{j}+1\right) b_{j+1} \ldots b_{n-r}$. Since $w$ has a minus cliff at $j$, we have $-b_{j}+b_{j-1}=\left|b_{j}\right|-\left|b_{j-1}\right| \geq 2$, therefore $w^{\prime} \in S(n, r)$ because $0 \geq b_{j-1} \geq b_{j}+2>b_{j}+1>b_{j}>b_{j+1}$ and $\left\|w^{\prime}\right\|=d$ since $b_{j} \leq-2$ implies $b_{j}+1<0$. As in the Case 1 , we note that $w^{\prime}$ covers $w$ in $S(n, d, r)$ because they differ between them only for a grain in the place $j$.

(ii) As in (i), we take $w=a_{r} \ldots a_{1} \mid b_{1} \ldots b_{n-r} \in S(n, d, r)$ and $a_{r+1}=r+1$ (the invisible pile in the place $r+1$ ). Let $w^{\prime \prime}=$ $a_{r}^{\prime \prime} \ldots a_{1}^{\prime \prime} \mid b_{1}^{\prime \prime} \ldots b_{n-r}^{\prime \prime}$ a generic element of $S(n, d, r)$ such that $w^{\prime \prime} \sqsupseteq w$ and $w^{\prime \prime} \neq w$. If we show that there exists an element $w^{\prime}=a_{r}^{\prime} \ldots a_{1}^{\prime} \mid b_{1}^{\prime} \ldots b_{n-r}^{\prime}$ of $S(n, d, r)$ such that $w^{\prime}=w \rightarrow^{k}$ for some $k=1,2,3$ and $w^{\prime \prime} \sqsupseteq w^{\prime}$ we complete the proof. Since $w^{\prime \prime} \neq w$, there is a place where the corresponding component of $w^{\prime \prime}$ is an integer strictly bigger than the integer component of $w$ corresponding to the same place. We distinguish several cases.

Case A. $a_{i}^{\prime \prime}>a_{i}$ for some $i \in\{r-1, \ldots, 1\}$.

Subcase A1. $a_{i+1} \geq a_{i}+2$. In this case we apply $R_{1}$ in the place $i$ to obtain $w^{\prime}=w \rightarrow{ }^{1}$ such that $w^{\prime \prime} \sqsupseteq w^{\prime}$.

Subcase A2. $a_{i+1}=a_{i}+1$. Since $a_{i}^{\prime \prime}>0$ and $i \leq r-1$, then $a_{i+1}^{\prime \prime}>a_{i}^{\prime \prime}>a_{i}$, therefore $a_{i+1}^{\prime \prime} \geq a_{i}+2>a_{i+1}$. Now, if $i+1=r$, then $r \geq a_{r}^{\prime \prime}>a_{r}$ and we can apply $R_{1}$ in the place $r$. We can assume therefore $i+1<r$. If $a_{i+2} \geq a_{i+1}+2$ we proceed as in A1 with $R_{1}$ in the place $i+1$, otherwise, if $a_{i+2}=a_{i+1}+1$ and $i+2=r$ we proceed as before with $R_{1}$ in the place $r$. Iterating, it follows that the cases to be examined are $a_{i+k} \geq a_{i+k-1}+2$ or $a_{i+k}=a_{i+k-1}+1$ and $i+k=r$. In all these case we can apply $R_{1}$ in the place $i+k-1$.

Case B. $a_{r}^{\prime \prime}>a_{r}$. In this case we just apply $R_{1}$ in the place $r$.

Case C. $b_{j}^{\prime \prime}>b_{j}$ for some $j \in\{1, \ldots, n-r\}$.

Subcase $C 1 . b_{j}^{\prime \prime}<0$. Then $b_{j} \geq-2$ and we can apply $R_{3}$ in the place $j$.

Subcase $C 2 . b_{j}^{\prime \prime}=0$ and $b_{j} \geq-2$. We apply $R_{3}$ in the place $j$.

Subcase C $3 . b_{j}^{\prime \prime}=0, b_{j}=-1$ and $a_{i}=1$ for some $i \in\{1, \ldots, r\}$. If $a_{1}=1$ then $w=r(r-1) \ldots 21 \mid 0 \ldots 0(-1) b_{j+1} \ldots b_{n-r}$ with $\|w\|=d$, therefore we can not have $w^{\prime \prime} \in S(n, d, r)$ such that $w^{\prime \prime} \sqsubseteq w$ and $w \neq w^{\prime \prime}$. We can assume then $i>1$, and therefore $a_{i-1}=\cdots=a_{1}=0$. Since $\left\|w^{\prime \prime}\right\|=\|w\|=d$, it must be $a_{i}^{\prime \prime}>a_{i-1}^{\prime \prime}>0$, hence $a_{i}^{\prime \prime} \geq 2$ and $a_{i}=1$. Now, if $a_{i+1} \geq a_{i}+2$, we apply $R_{1}$ in the place $i$. We can suppose then that $a_{i+1}=a_{i}+1=2$. Since $a_{i-1}^{\prime \prime}>0$, it must be $a_{i+2}^{\prime \prime} \geq 4$, therefore, if $a_{i+2}=a_{i+2}^{\prime \prime}$ then $a_{i+2} \geq a_{i+1}+2$ and we can apply $R_{1}$ in the place $i+1$. Hence we can assume $a_{i+2}<a_{i+2}^{\prime \prime}$. If $a_{i+2} \geq a_{i+1}+2$ we apply then $R_{1}$ in the place $i+1$ because $a_{i+1}^{\prime \prime} \geq 3>a_{i+1}=2$. Then we can suppose $a_{i+2}=a_{i+1}+1=3$. Iterating this reasoning, we apply $R_{1}$ in some place $i+k$, with $k \geq 1$, or we obtain for $w$ and $w^{\prime \prime}$ the following forms:

$$
\begin{aligned}
& w=l(l-1) \ldots 3210 \ldots 0 \mid 0 \ldots 0(-1) b_{j+1} \ldots b_{n-r}, \\
& w^{\prime \prime}=l^{\prime}\left(l^{\prime}-1\right) \ldots 3210 \ldots 0 \mid 0 \ldots 00 b_{j+1}^{\prime \prime} \ldots b_{n-r}^{\prime \prime}
\end{aligned}
$$

with $r \geq l^{\prime}>l$. We can apply therefore $R_{1}$ in the place $r$.

Subcase C4. $b_{j}^{\prime \prime}=0, b_{j}=-1$ and $a_{i} \neq 1$ for each $i \in\{1, \ldots, r\}$.

In this case there is at least one place $i \in\{1, \ldots, r\}$ such that $a_{i}=0$. We take this $i$ maximal, so that $i=r$ or $i<r$ and 
$a_{i+1}>0$. Then, since $b_{j}^{\prime \prime}=0, b_{j}=-1, a_{i+1}^{\prime \prime} \geq a_{i+1}>0$ and $\|w\|=\left\|w^{\prime \prime}\right\|=d$, it must be necessarily $a_{i}^{\prime \prime}>0$. We apply then $R_{2}$ shifting the negative grain from the place $j$ into a positive grain in the place $i$, that is, we take $w^{\prime}=w \rightarrow{ }^{2}$ with $a_{i}^{\prime}=1$ and $b_{j}^{\prime}=0$ and all other components unchanged.

Hence in all the previous cases we obtain an element $w^{\prime}=$ $w \rightarrow{ }^{k}$, for some $k=1,2,3$, such that $w^{\prime} \sqsubseteq w^{\prime \prime}$.

Below we draw the Hasse diagram of the lattice $S(5,3,2)$ by using the evolution rules $R_{1}, R_{2}, R_{3}$ starting to the minimum element of this lattice, which is $00 \mid 123$. We label a generic edge of the next diagram with the integer $k$ if it leads to a production that uses $R_{k}$, for $k \in\{1,2,3\}$ :

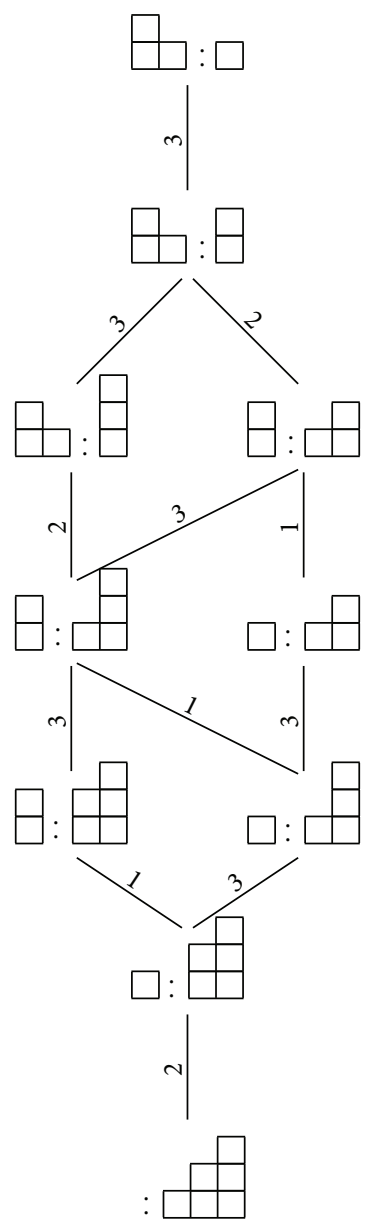

Since $S(n, d, r)$ is a finite distributive lattice it is also graded; in the next proposition we determine its rank function.

Proposition 4. The rank function $\rho$ of $S(n, d, r)$ is $\rho(w)=$ $\sum(w)-\sum(\widehat{0})-\left(|w|_{>}-|\widehat{0}|_{>}\right)$.

Proof. We denote by $\varrho$ the rank function of the graded lattice $S(n, r)$. It is easy to verify that $\rho(w)=\sum(w)-\sum(\widehat{0})$ for each $w \in S(n, r)$ (see also [22]). Let $w>w_{t}>\cdots>w_{1}>\widehat{0}$ be any saturated chain from $\widehat{0}$ to $w$. Let us assume that in this chain $w$ is obtained from $\widehat{0}$ with $k$ applications of $R_{2}$, for some integer $k \geq 0$. To each step $l \in\{1, \ldots, t\}$ where we apply $R_{2}$, there is the following situation: $w_{l} \sqsupset u_{l} \sqsupset w_{l-1}$, for exactly one only element $u_{l} \in S(n, r) \backslash S(n, d, r)$. This means that $\varrho(w)=$ $(t+1)+k$, that is, $\rho(w)=\varrho(w)-k$. The integer $k$ is also the difference between the number of positive parts of $w$ and the number of positive parts of $\widehat{0}$. Hence the thesis follows.

In the next proposition we compute the rank of the lattice $S(n, d, r)$.

Proposition 5. $\operatorname{rank}(S(n, d, r)=d(n-d)$.

Proof. If $r \geq d>n-r$, then $\hat{1}=r \ldots(r-d+1) 0 \ldots 0 \mid 0 \ldots 0$ and

$$
\widehat{0}=(d-(n-r)) \ldots 10 \ldots 0 \mid(-1)(-2) \ldots(-(n-r)) .
$$

So that $|\widehat{1}|_{>}-|\widehat{0}|_{>}=n-r$. If $d>n-r$ and $d>r$, then $\widehat{1}=r \ldots 1 \mid 0 \ldots 01 \ldots(d-r)$ and $\widehat{0}=(d-(n-r)) \ldots 10 \ldots 0 \mid$ $(-1)(-2) \ldots(-(n-r))$. So that $|\hat{1}|_{>}-|\hat{0}|_{>}=n-d$. If $d \leq$ $n-r$ and $d \leq r$, then $\hat{1}=r \ldots(r-d+1) 0 \ldots 0 \mid 0 \ldots 0$ and $\hat{0}=0 \ldots 0 \mid 0 \ldots 0(-((n-r)-(d-1))) \ldots(-(n-r))$. Therefore $|\widehat{1}|_{>}-|\widehat{0}|_{>}=d$. Finally, if $d \leq n-r$ and $d>r$, then $\widehat{1}=r \ldots 1 \mid 0 \ldots 0(-1) \ldots(-(d-r))$ and $\widehat{0}=0 \ldots 0 \mid$ $0 \ldots 0(-((n-r)-(d-1))) \ldots(-(n-r))$. Therefore $|\widehat{1}|_{>}-|\widehat{0}|_{>}=r$. The rank of $S(n, d, r)$ is obviously $\rho(\widehat{1})-\rho(\widehat{0})$, that we compute in all the previous cases applying the Proposition 4 . The thesis follows then from simply arithmetic manipulations.

\section{Dynamics of $S(n, d, r)$ as Sand Piles Model}

In this section we study the lattice $S(n, d, r)$ as a discrete dynamical system. For the terminology concerning the discrete dynamical system we refer to [21]. In such a context, we call configuration a generic element of $S(n, d, r)$. The initial configuration is $\widehat{0}$. Each configuration converges, in sequential and in parallel, toward the unique fixed point $\hat{1}$ because of the lattice structure of the model. Let us note that if $w$ is a configuration, when we use the evolution rules in parallel, on each column of $w$ we can apply (due to the nature of the Rules $R_{1}, R_{2}, R_{3}$ ) exactly one evolution rule, hence our model is deterministic. With the same notations of [3], we denote respectively by $T_{\text {sec }}(w)$ and $T_{\text {par }}(w)$ the number of time steps required to reach $\hat{1}$ starting from the configuration $w$, using the sequential or the parallel updating scheme. Obviously $T_{\text {sec }}(w)$ is independent of the order in which the sites are updated because $S(n, d, r)$ is a graded lattice. Moreover it is also clear that

$$
T_{\text {sec }}(w)=\rho(\widehat{1})-\rho(w) .
$$

We study now some properties of the dynamics in parallel. If $w$ and $w^{\prime}$ are two different configurations, we say that $w^{\prime}$ is a parallel successor of $w$, and we write $w \rightrightarrows w^{\prime}$ or $w^{\prime}=w \rightrightarrows$, if $w^{\prime}$ is the configuration which is obtained with all the possible parallel applications of the Rules $1-3$ on the parts of $w$. If we can apply in parallel $m_{i}$ times $R_{i}$ on $w$, for $i=1,2,3$, we set $M(w):=\left(m_{1}, m_{2}, m_{3}\right)$ and $|M(w)|:=m_{1}+m_{2}+m_{3}$. Let us note that $m_{2}$ can be only 0 or 1 . Obviously there is a unique finite sequence $\left(w_{0}, w_{1}, \ldots, w_{s}\right)$ of configurations such that

$$
w_{0}=\widehat{0} \rightrightarrows w_{1} \rightrightarrows \cdots \rightrightarrows w_{s-1} \rightrightarrows w_{s}=\widehat{1} .
$$


The sequence in (11) is obviously a chain of length $s$ in $(S(n, d, r), \sqsubseteq)$ that we call fundamental chain of $S(n, d, r)$. It is clear that $T_{\text {par }}(\widehat{0})=s$. We also call fundamental sequence of $S(n, d, r)$ the finite integer sequence

$$
\left(\left|M\left(w_{0}\right)\right|,\left|M\left(w_{1}\right)\right|, \ldots,\left|M\left(w_{s-1}\right)\right|\right) .
$$

Remark 6. If $\left(w_{0}, w_{1}, \ldots, w_{s}\right)$ is the fundamental chain of $S(n, d, r)$ then

$$
\sum_{i=1}^{s-1}\left|M\left(w_{i}\right)\right|=\operatorname{rank}(S(n, d, r)) .
$$

In the next result we compute the exact value of $T_{\text {par }}(\widehat{0})$ for a wide range of the integers parameters $n, d, r$ and in another case we provide a lower estimate.

Theorem 7. (i) If $d=(n-r)=r, T_{p a r}(\widehat{0})=2 d-1$.

(ii) If $d=(n-r)<r, T_{\text {par }}(\widehat{0})=d+r-1=n-1$.

(iii) If $d<(n-r)$ and $r \geq d, T_{\text {par }}(\widehat{0}) \geq 2 d-2$.

(iv) If $r>d>(n-r)$ and $2 d=n, T_{\text {par }}(\widehat{0})=2 d-1$.

(v) If $r>d>(n-r)$ and $2 d-n<0, T_{\text {par }}(\widehat{0})=n-1$.

Moreover, in all the previous cases the fundamental sequence of $S(n, d, r)$ is symmetric and unimodal.

Proof. (i) If $d=(n-r)=r$ then $\widehat{0}=0 \ldots 0 \mid 1 \ldots(n-r)$ and $\widehat{1}=r \cdots 1 \mid 0 \cdots 0$. In this case the first and the last rule which applies is always $R_{2}$ : in between all the rules $R_{1}, R_{2}, R_{3}$ apply in a very symmetric way, in view of their definition and of the symmetry of three parameters of $S(n, d, r)$. The number of rules which apply at each step follows this sequence: $1, \ldots,(d-1), d,(d-1), \ldots, 1$ and the unimodularity and the symmetry of the sequence of parallel configurations is therefore straightforward. Moreover there exists a unique time $T=d$ in which the maximal number $d$ of rules apply; the string at which the $d$ rules apply is always of the following type:

if $d$ is even: $(d-1) \ldots 1 \ldots 0 \mid 0 \ldots 2 \ldots d$, where in both positive and negative part each number is the previous minus 2;

if $d$ is odd: $(d-1) \ldots 2 \ldots 0 \mid 0 \ldots 1 \ldots d$, where in both positive and negative part each number is the previous minus 2 .

Hence $T_{\text {par }}(\widehat{0})=2 d-1$.

(ii) If $d=(n-r)<r$ then $\widehat{0}=0 \ldots 0 \mid 1 \ldots(n-r)$ and $\widehat{1}=r \ldots(r-d+1) \ldots 0 \mid 0 \ldots 0$. The first rule which applies is always $R_{2}$ and the last one is always $R_{1}$. In this case there are several strings obtained with the maximal number of rules which is $d$ : the first one which appears from the bottom in the fundamental sequence of $S(n, d, r)$ is:

if $d$ is even, $(d-1)(d-3) \ldots 10 \ldots 0 \mid 0 \ldots 2 \ldots d$, where in both positive and negative part each number is the previous minus 2;

if $d$ is odd, $(d-1)(d-3) \ldots 20 \ldots 0 \mid 0 \ldots 1 \ldots(d-2) d$, where in both positive and negative part each number is the previous minus 2 .
The number of steps with $d$ rules is exactly $(r-d+1)$ and the number of rules which apply at each step follows this sequence: $1, \ldots,(d-1), d, \ldots d,(d-1), \ldots, 1$ : hence the unimodularity and the symmetry of the sequence of the parallel configurations is straightforward. Finally, $T_{\text {par }}(\widehat{0})=$ $d+r-1=n-1$.

(iii) With these parameters, $\widehat{0}=0 \ldots 0 \mid 0 \ldots(n-r-$ $d+1) \ldots(n-r)$ and $\hat{1}=r \ldots(r-d+1) 0 \ldots 0 \mid 0 \ldots 0$. The first rule from the bottom which applies is always $R_{3}$ and the last rule from the top which applies is always $R_{1}$. In this case there are several strings obtained with the maximal number of rules which is still $d$, and the number of rules which apply at each step follows this sequence: $1, \ldots,(d-1), d, \ldots d,(d-$ $1), \ldots, 1$ : therefore the unimodularity and the symmetry of the sequence of the parallel configurations follow such as the lower bound for $T_{\text {par }}(\widehat{0})$.

(iv) With these parameters, $\widehat{0}=(d-n+r) \cdots 1 \cdots 0$ । $1 \ldots(n-r)$ and $\widehat{1}=r \cdots(r-d+1) \cdots 0 \mid 0 \cdots 0$. In this case the first and the last rule which applies is always $R_{1}$ : in between all the rules $R_{1}, R_{2}, R_{3}$. The number of rules which apply at each step follows this sequence: $1, \ldots,(d-1), d,(d-1), \ldots, 1$ and the unimodularity and the symmetry of the sequence of parallel configurations is immediate. Moreover there exists a unique time $T=d$ in which the maximal number $d$ of rules apply. Hence $T_{\text {par }}(\widehat{0})=2 d-1$.

(v) With these parameters, $\hat{0}=(d-n+r) \cdots 1 \cdots 0$ । $1 \ldots(n-r)$ and $\hat{1}=r \cdots(r-d+1) \cdots 0 \mid 0 \cdots 0$. In this case the first and the last rule which applies is always $R_{1}$. A difference with the previous case is that here there are several strings obtained with the maximal number of rules which is $d$; the number of string at which $d$ rules apply is exactly $(n-2 d+1)$. The very interesting thing is that $T_{\text {par }}(\widehat{0})=n-1$ is independent of $d$ in this case, as in case (ii). Obviously also in this case we have the unimodularity and the symmetry of the sequence of the parallel configurations.

\section{References}

[1] T. Brylawski, “The lattice of integer partitions," Discrete Mathematics, vol. 6, no. 3, pp. 201-219, 1973.

[2] E. Goles, "Sand pile automata," Annales de l'Institut Henri Poincaré, vol. 56, no. 115, pp. 75-90, 1992.

[3] E. Goles and M. A. Kiwi, "Games on line graphs and sand piles," Theoretical Computer Science, vol. 115, no. 2, pp. 321-349, 1993.

[4] J. Spencer, "Balancing vectors in the max norm," Combinatorica, vol. 6, no. 1, pp. 55-65, 1986.

[5] P. Bak, C. Tang, and K. Wiesenfeld, "Self-organized criticality," Physical Review A, vol. 38, no. 1, pp. 364-374, 1988.

[6] D. Dhar, "The Abelian sandpile and related models," Physica A, vol. 263, no. 1-4, pp. 4-25, 1999.

[7] E. Goles and M. Margenstern, "Universality of the chip-firing game," Theoretical Computer Science, vol. 172, no. 1-2, pp. 121-134, 1997.

[8] E. Goles, M. Morvan, and H. Phan, "Lattice structure and convergence of a game of cards," Annals of Combinatorics, vol. 6, pp. 327-335, 2002.

[9] J. Cervelle, E. Formenti, and B. Masson, "From sandpiles to sand automata," Theoretical Computer Science, vol. 381, no. 1-3, pp. $1-28,2007$. 
[10] E. Goles and M. A. Kiwi, One-Dimensional Sand Piles, Cellular Automata and Related Models, Nonlinear Phenomena in Uids, Solids and Other Complex Systems, 1990.

[11] E. Formenti, B. Masson, and T. Pisokas, "On symmetric sandpiles," Lecture Notes in Computer Science, vol. 4173, pp. 676-685, 2006.

[12] E. Formenti, B. Masson, and T. Pisokas, "Advances in symmetric sandpiles," Fundamenta Informaticae, vol. 76, no. 1-2, pp. 91-112, 2007.

[13] E. Goles, M. Morvan, and H. D. Phan, "Sandpiles and order structure of integer partitions," Discrete Applied Mathematics, vol. 117 , no. $1-3$, pp. 51-64, 2002.

[14] M. H. Le and T. H. D. Phan, "Strict partitions and discrete dynamical systems," Theoretical Computer Science, vol. 389, no. 1-2, pp. 82-90, 2007.

[15] M. Latapy, "Partitions of an integer into powers," in Proceedings of the conference Discrete Models: Combinatorics, Computation, and Geometry, Discrete Mathematics and Theoretical Computer Science, pp. 215-228, 2001.

[16] M. Latapy, "Integer partitions, tilings of 2D-gons and lattices," Theoretical Informatics and Applications, vol. 36, no. 4, pp. 389-399, 2002.

[17] M. Latapy, R. Mantaci, M. Morvan, and H. D. Phan, "Structure of some sand piles model," Theoretical Computer Science, vol. 262, no. 1-2, pp. 525-556, 2001.

[18] M. Latapy and H. D. Phan, "The lattice structure of chip firing games and related models," Physica D, vol. 155, no. 1-2, pp. 69-82, 2001.

[19] M. Latapy and T. H. D. Phan, "The lattice of integer partitions and its infinite extension," Discrete Mathematics, vol. 309, no. 6, pp. 1357-1367, 2009.

[20] P. T. H. Duong and T. T. T. Huong, "On the stability of sand piles model," Theoretical Computer Science, vol. 411, no. 3, pp. 594-601, 2010.

[21] E. Goles, M. Latapy, C. Magnien, M. Morvan, and H. D. Phan, "Sandpile models and lattices: a comprehensive survey," Theoretical Computer Science, vol. 322, no. 2, pp. 383-407, 2004.

[22] C. Bisi and G. Chiaselotti, "A class of lattices and boolean functions related to the Manickam-Miklös-Singhi Conjecture," Advances in Geometry, vol. 13, no. 1, pp. 1-27, 2013.

[23] G. Chiaselotti, "On a problem concerning the weight functions," European Journal of Combinatorics, vol. 23, no. 1, pp. 15-22, 2002.

[24] G. Chiaselotti, G. Infante, and G. Marino, "New results related to a conjecture of Manickam and Singhi," European Journal of Combinatorics, vol. 29, no. 2, pp. 361-368, 2008.

[25] G. Chiaselotti, G. Marino, and C. Nardi, "A minimum problem for chcnite sets of real numbers with nonnegative sum," Journal of Applied Mathematics, vol. 2012, Article ID 847958, 15 pages, 2012.

[26] G. Marino and G. Chiaselotti, "A method to count the positive 3 -subsets in a set of real numbers with non-negative sum," European Journal of Combinatorics, vol. 23, no. 5, pp. 619-629, 2002.

[27] K. Engel and C. Nardi, "Solution of a problem on non-negative subset sums," European Journal of Combinatorics, vol. 33, pp. 1253-1256, 2012.

[28] R. P. Stanley, "Weyl groups, the hard Lefschetz theorem, and the Sperner property," SIAM Journal on Algebraic and Discrete Methods, vol. 1, pp. 168-184, 1980.
[29] S. Bezrukov and K. Engel, Properties of Graded Posets Preseved by Some Operations, Algorithms and Combinatorics, vol. 14 of The mathematics of Paul Erdos, Springer, Berlin, Germany, 1997.

[30] G. E. Andrews, "Euler's "de partitio numerorum"', Bulletin of the American Mathematical Society, vol. 44, no. 4, pp. 561-573, 2007.

[31] W. J. Keith, "A bijective toolkit for signed partitions," Annals of Combinatorics, vol. 15, no. 1, pp. 95-117, 2011.

[32] K. J. Al-Agha and R. J. Greechie, "The involutory dimension of involution posets," Order, vol. 18, no. 4, pp. 323-337, 2001.

[33] K. Brenneman, R. Haas, and A. G. Helminck, "Implementing an algorithm for the twisted involution poset for Weyl groups," in Proceedings of the Thirty-Seventh Southeastern International Conference on Combinatorics, Graph Theory and 7-Computing, vol. 182, pp. 137-144, 2006. 


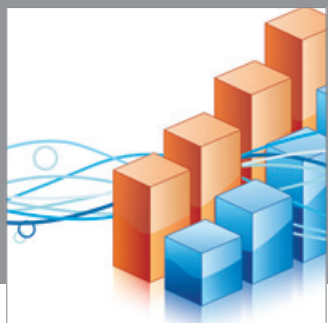

Advances in

Operations Research

mansans

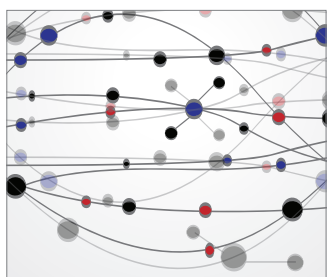

The Scientific World Journal
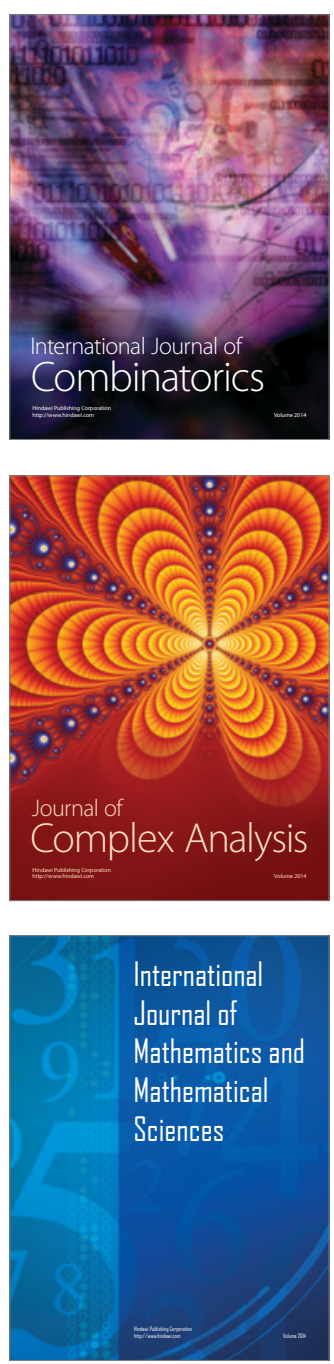
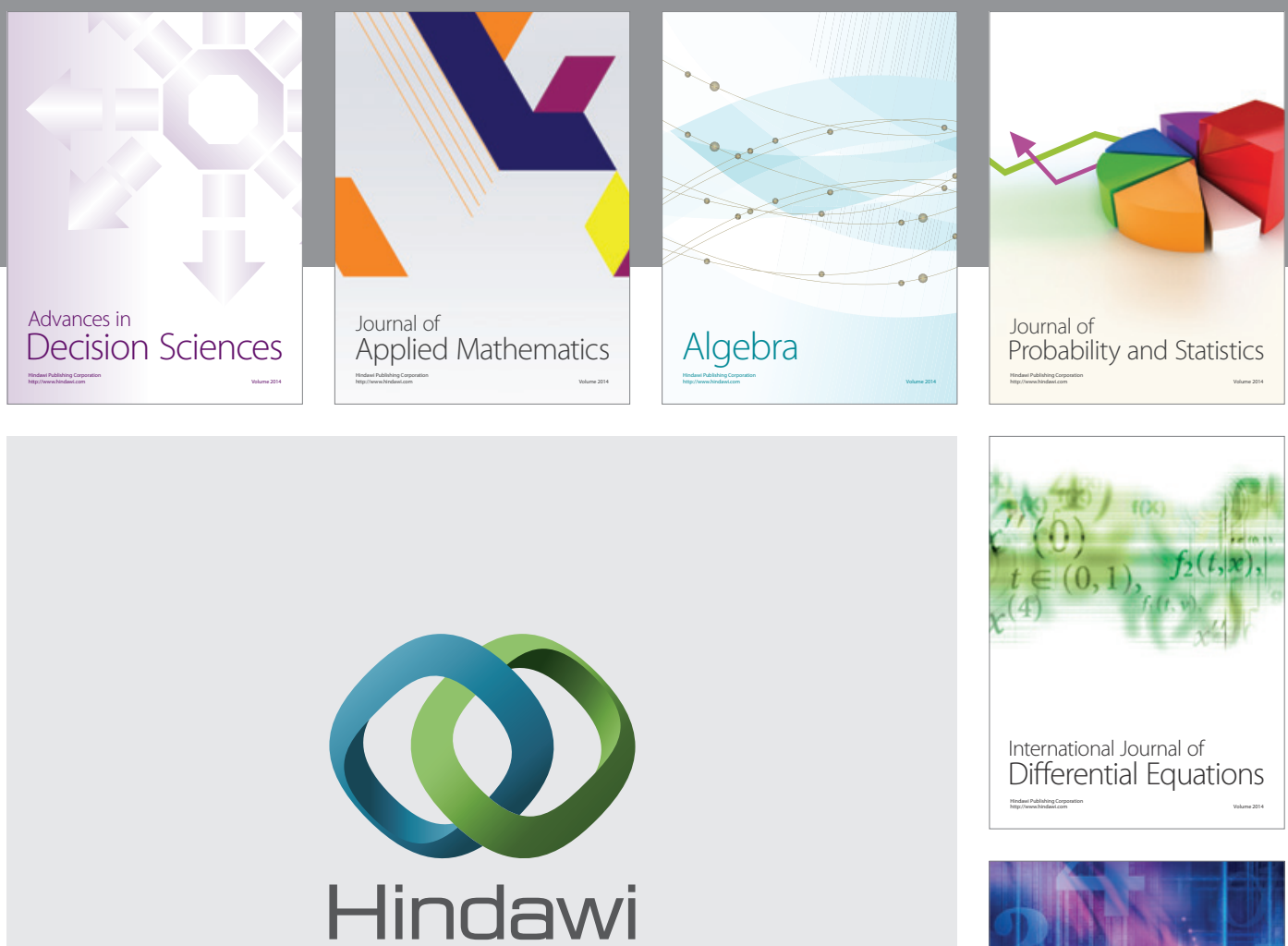

Submit your manuscripts at http://www.hindawi.com
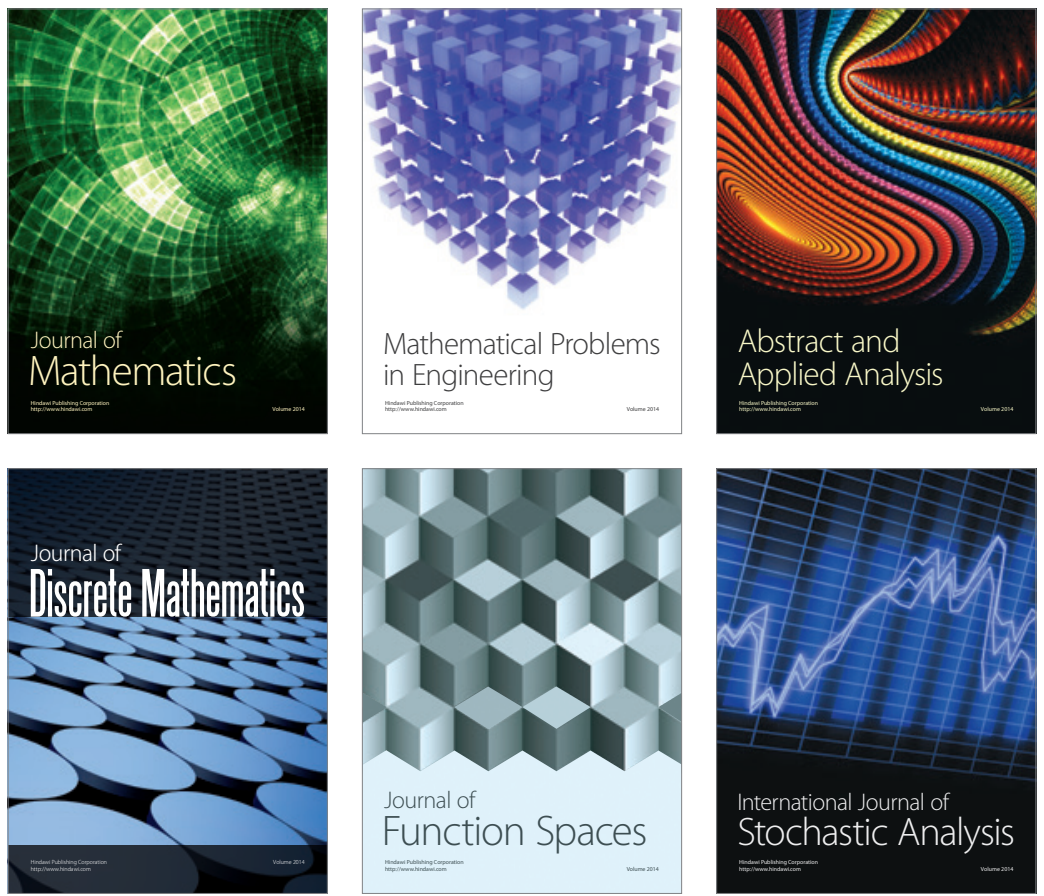

Journal of

Function Spaces

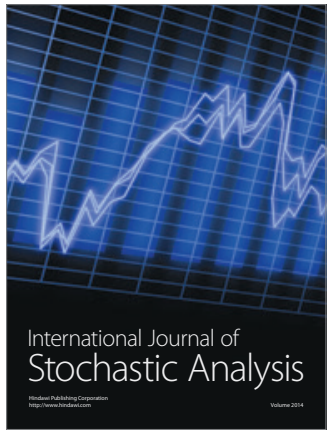

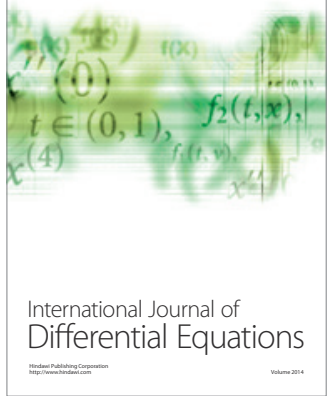
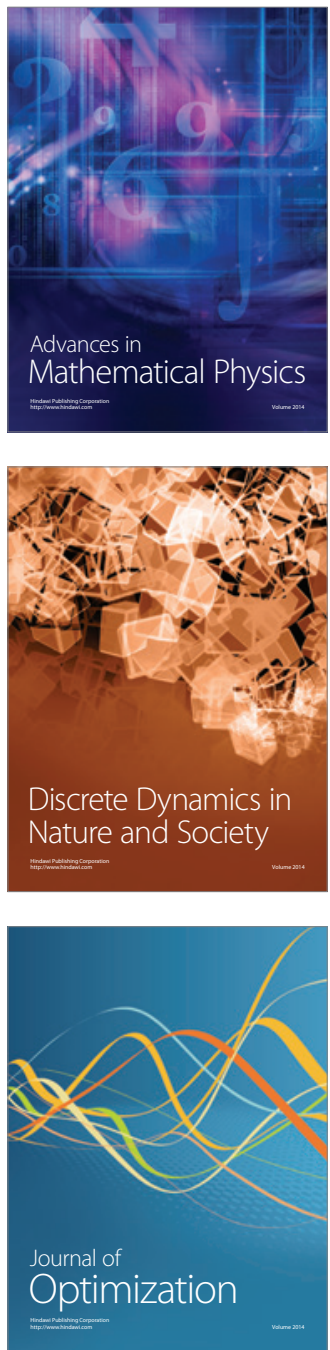